On analytical formulae for navigation lock filling-emptying and overtravel

LAURENT SCHINDFESSEL, Ph.D. student, Hydraulics Laboratory, Department of Civil Engineering, Ghent University, Sint-Pietersnieuwstraat 41 B5, B-9000 Ghent, Belgium

Email: Laurent.Schindfessel@UGent.be (author for correspondence)

TOM DE MULDER (IAHR Member), Associate professor, Hydraulics Laboratory, Department of Civil Engineering, Ghent University, Sint-Pietersnieuwstraat 41 B5, B-9000 Ghent, Belgium

Email:TomFO.DeMulder@UGent.be

STEPHAN CREELLE, Ph.D. student, Hydraulics Laboratory, Department of Civil Engineering, Ghent University, Sint-Pietersnieuwstraat 41 B5, B-9000 Ghent, Belgium Email:Stephan.Creelle@UGent.be

GERALD A. SCHOHL (IAHR Member), Senior hydraulic expert, Retired from Tennessee Valley Authority Engineering Laboratory, Hydraulic Consultant, Knoxville, Tennessee Email:jschohl@usit.net

Running Head: Navigation lock filling-emptying and overtravel formulae 


\title{
On analytical formulae for navigation lock filling-emptying and overtravel
}

\begin{abstract}
In culvert-based navigation lock filling-emptying systems, inertia effects have a significant influence on the filling-emptying time and cause a (damped) oscillation of the water surface in the lock chamber around its equalization level, referred to as the overtravel phenomenon. In this paper, the derivation of analytical formulae for the lock filling-emptying time and overtravel peak of systems consisting of a number of identical culverts is revisited. In comparison to earlier publications, the underlying assumptions are made explicit and the importance of accounting for the surface area ratio of lock chamber to upper reservoir in case of filling (resp. lower reservoir in case of emptying) is pointed out. Additionally, it is shown how the applicability of the analytical formulae can be extended to lock filling-emptying systems with more complex lay-outs by using an "equivalent culvert" approach. The validity of the analytical formulae is thoroughly assessed, first by comparing to an accurate numerical solution of the governing non-linear second-order differential equations, and secondly, by means of experiments in a physical model.
\end{abstract}

Keywords: culvert; filling time; inertia; navigation lock; overtravel

\section{Introduction}

Several types of navigation lock filling and emptying systems exist, many of which make use of culverts to transfer water by gravity from an upper reservoir to the lock chamber in case of filling, or from the lock chamber to a lower reservoir in case of emptying, see e.g. PIANC (1986). In a system with bypass (or looped) culverts in the lock head, the culverts are relatively short in comparison to the lock chamber length, whereas in a sidewall filling system with ports, the longitudinal culverts are relatively long. Various other types of culvert-based systems exist, making use of different combinations of longitudinal culverts (in the sidewalls or central in the chamber) and lateral culverts on top of or under the lock chamber floor.

In the hydraulic design of a navigation lock filling and emptying system, the lock filling or emptying time is a key parameter, which should remain below a specified threshold value (without causing an inconvenient level of turbulence in the lock chamber). Besides numerical modelling and physical modelling, also analytical formulae are still useful as design tools nowadays, especially in the early stages of design.

For a simple through-the-gate system consisting of valves in the lock gates - i.e. not making use of culverts - an analytical formula for the filling (resp. emptying) time can be derived in a straightforward way. This classical formula has been, and still is, also applied to culvert-based systems. 
For a culvert-based system in a lock of the Panama Canal, however, it was noted in Pillsbury (1915) that lock filling does not exactly follow the classical formula and that, at the end of filling the water level in the lock chamber tends to rise above the level of the upper reservoir. For emptying, an analogous behaviour is observed. This phenomenon is referred to as overtravel and is characterized by a damped oscillation of the water surface in the lock chamber with respect to its equalization level, as is schematically shown in Fig. 1. Pillsbury (1915) attributed these features to the inertia of the water flowing in the culverts and derived an approximate formula for the lock filling (or emptying) time, containing the height of the first overtravel peak as a parameter. The Pillsbury formula appears e.g. in Dehousse (1985) and USACE (2006). The latter reference also provides, without explicit derivation, an analytical formula for the height of the first overtravel peak. Dehousse (1985) on the other hand, does provide a derivation for the height of the first three overtravel peaks.

In section 2 of this paper, the derivation of the aforementioned analytical formulae will be revisited, in order to clarify the underlying assumptions and approximations, and possibly extend their scope. It will turn out that these formulae assume a lock filling (and emptying) system consisting of a number of identical culverts.

The governing equation is a non-linear second-order differential equation, containing a quadratic damping term (section 2.1). Such an equation is generic to many problems in hydraulics. It describes e.g. also the water level behaviour in a surge tank of a hydropower conveyance system. In the latter field of application, the overtravel phenomenon and its governing equation have already been studied to some depth; see e.g. Jaeger (1956), Reisman \& Silvers (1967) and Steber \& Reisman (1969). This is beneficial for the study of overtravel in navigation lock filling, allowing some insight and modelling approaches to be shared. Yet, navigation lock filling and overtravel deserves a study in itself, given the large ratio of chamber surface area to cross-sectional area of the feeding culvert and the different initial conditions, as compared to surge tanks.

Even when the damping parameter is assumed to be a constant, no exact closed form solution of the governing differential equation is known to date. In section 2.2, an approximate solution of the complete equation will be derived, in a similar way as proposed by Pillsbury (1915), yielding analytical formulae for the lock filling time, the height of the first overtravel peak and a typical frequency of the water level oscillations around the equalization level. The formulae derived in this paper will also apply to lock filling (or emptying) configurations in which the surface area of the upper (or lower) reservoir is not infinitely large in comparison to the one of the lock chamber, an assumption which is often implicitly made in earlier publications. Some alternative analytical formulae will be derived in section 2.3 as exact solutions to simplified forms of the governing equation. A numerical 
validation of the derived analytical formulae will be carried out in section 2.4 , based on a comparison with an accurate numerical solution of the complete governing equation.

In section 3 of this paper, it will be shown how the applicability of the analytical formulae can be extended to culvert-based systems with a more complex lay-out, based on a so-called equivalent culvert approach. An experimental validation of the analytical formulae, including the equivalent culvert approach, is carried out in section 4, making use of a physical model. Finally, conclusions are provided in section 5.

\section{Modelling the case of lock filling through $\boldsymbol{n}$ identical culverts}

\subsection{Governing equation}

In this paper, only filling of a navigation lock will be considered, since lock emptying is completely analogous. Consider the situation depicted in Fig. 2. A navigation lock with a chamber surface area $S_{c}$ is filled from an upper reservoir having a surface area $S_{u}$ by means of $n$ identical culverts. Each culvert has a constant cross-sectional area $A$, a length $L$, a constant total head loss coefficient $k$ and a (time-dependent) flow rate $Q$. A positive flow rate is associated with a flow in the direction of the lock chamber.

Water levels are described by means of a $z$-axis, pointing vertically upwards with an arbitrary origin, e.g. at the floor of the lock chamber. The time-dependent water level in the lock chamber is denoted by $z_{c}$. Depending on the lock configuration (cf. infra), the water level in the upper reservoir $z_{u}$ might be constant or might vary in time. Let $z_{e}$ be the equalization water level, which is assumed to be independent of time. The lift $\ell$ is then defined as $\ell=z_{e}-z_{o}$, where $z_{o}$ denotes the initial water level in the lock chamber.

Prior to deriving the governing equation for lock filling, some assumptions are made: (i) the water surfaces in the chamber and the upper reservoir are horizontal at every instant in time, i.e. translational waves are neglected, (ii) the chamber and the upper reservoir are prismatic, i.e. their surface areas do not change with the water level, (iii) drying of the chamber or the upper reservoir is excluded, (iv) the valves in the culverts are opened instantaneously, (v) the only source of inertia is the flow in the culverts, i.e. the inertia in the lock chamber and in the upper reservoir is neglected, and (vi) the sum of all frictional and minor head losses in a culvert is characterized by a constant total head loss coefficient, i.e. the Reynolds number dependency (hence the time-dependency) of the friction factor is neglected.

Combining the unsteady energy equation for a culvert,

$$
\left(z_{u}-z_{c}\right)=\frac{k}{2 g A^{2}}|Q| Q+\frac{L}{g A} Q^{\prime}
$$

with the continuity equation of the lock chamber, 


$$
S_{c} z_{c}^{\prime}=n Q
$$

and the mass balance between the upper reservoir and the chamber,

$$
\left(z_{u}-z_{e}\right) S_{u}=\left(z_{e}-z_{c}\right) S_{c}
$$

yields the following second-order differential equation:

$$
\left(1+\frac{S_{c}}{S_{u}}\right)\left(z_{c}-z_{e}\right)+\frac{k}{2 g}\left(\frac{S_{c}}{n A}\right)^{2}\left|z_{c}^{\prime}\right| z_{c}^{\prime}+\frac{L}{g}\left(\frac{S_{c}}{n A}\right) z_{c}^{\prime \prime}=0
$$

where the prime denotes a derivative with respect to time $t$. The initial conditions are:

$$
z_{c}=z_{o}\left(=z_{e}-\ell\right) \text { and } z_{c}{ }^{\prime}=0 \text { at } t=0
$$

Note that Eq. (3) expresses that the upper reservoir and the lock chamber act as communicating vessels. As a consequence, the water level in the upper reservoir $z_{u}$ might vary in time because of filling of the lock chamber. All other causes of time-dependency of $z_{u}$ (like e.g. tidal waves, translation waves, etc.) are neglected in this paper, in order for the equalization level $z_{e}$ to be constant in time.

By introducing an inverse time scale $\omega_{0}$,

$$
\omega_{\mathrm{o}}=\left(\frac{g n A}{L S_{c}}\right)^{\frac{1}{2}}
$$

a dimensionless time coordinate $\tau$ can be defined as $\tau=\omega_{0} t$. Similarly, the deviation of the (time-dependent) water surface in the lock chamber from its equalization level can be presented by the dimensionless parameter $\zeta$ :

$$
\zeta=\frac{z_{c}-z_{e}}{\ell}
$$

where $z_{c}$ is a function of time $t$, and $\zeta$ is a function of dimensionless time $\tau=\omega_{\mathrm{o}} t$.

Equation (4) can now be rewritten in dimensionless form:

$$
(1+\sigma) \zeta+\psi|\dot{\zeta}| \dot{\zeta}+\ddot{\zeta}=0
$$

where a dot represents a derivative with respect to $\tau, \sigma$ denotes the ratio of the surface areas, i.e. $\sigma=S_{c} / S_{u}$, while $\psi$ is a dimensionless damping parameter:

$$
\psi=\frac{k}{2} \frac{\ell}{L} \frac{S_{c}}{n A}
$$

In dimensionless form, the initial conditions of Eq. (5) become:

$$
\zeta=-1 \text { and } \dot{\zeta}=0 \text { at } \tau=0
$$

The parameter $\sigma$ is a positive constant that depends on the configuration of the navigation lock. If the surface area of the upper reservoir $S_{u}$ is infinitely large (in comparison to the surface area of the lock chamber $S_{c}$ ), then $\sigma=0$. This situation is typical for filling a lock chamber from a (long) river or canal reach (with a large forebay). The case of a staircase lock, where relatively short reaches are present between consecutive lock chambers, corresponds to the range $0<\sigma<1$. In case of a double-lift lock, both chambers have an equal surface area, and $\sigma$ equals 1. From Eq. (3) it follows that the case $\sigma=0$ is equivalent to $z_{u}$ 
being constant in time (and equal to the equalization level $z_{e}$ ). For all other values of the $\sigma$ parameter, lock filling will give rise to $z_{u}$ varying in time, though the equalization level $z_{e}$ is constant in time.

Unless stated otherwise, both damping and culvert inertia are taken into account in this paper. The corresponding non-linear second-order differential Eq. (8) is referred to as the complete governing equation, which needs to be solved in combination with Eq. (10). The governing equation is said to be simplified when either damping or inertia is neglected.

\subsection{Analytical formulae derived from approximate solution of complete equation}

Similar to other quadratically damped hydraulic systems mentioned in the introduction, no exact analytical solution of the governing equation (8) and initial conditions (10) seems possible when both damping and inertia are accounted for.

A first integration of Eq. (8) in the time interval that $\dot{\zeta}$ is positive (i.e. from the start of filling at $\tau=0$ till the moment that the first overtravel peak is reached at $\tau=\tau_{1}$ ) yields:

$$
(\dot{\zeta})^{2}=\frac{1+\sigma}{2 \psi^{2}}-\frac{1+\sigma}{\psi} \zeta-\left(\frac{1+\sigma}{2 \psi^{2}}+\frac{1+\sigma}{\psi}\right) \exp \{-2 \psi(\zeta+1)\}
$$

As was already conjectured by Pillsbury (1915) and will be demonstrated below, the exponential term in Eq. (11) can often be neglected in good approximation for many culvertbased lock filling-emptying systems. Further integration then yields:

$$
\zeta=\frac{1}{2 \psi}-\left[\left(\frac{1}{2 \psi}+1\right)^{\frac{1}{2}}-\frac{1}{2}\left(\frac{1+\sigma}{\psi}\right)^{\frac{1}{2}} \tau\right]^{2}
$$

Equation (12) allows derivation of approximate analytical formulae (see table 1, formulae with a Roman numeral I followed by a letter) for the most important navigation lock filling parameters (Fig. 1) in dimensionless form: (i) the filling time $\tau_{\text {full }}\left(=\omega_{\mathrm{o}} t_{\text {full }}\right)$, (ii) the water surface deviation from the equalization level at the first overtravel peak $\zeta_{1}\left(=\left(z_{1}-z_{e}\right) / \ell\right)$, (iii) the time of the first overtravel peak $\tau_{1}\left(=\omega_{0} t_{1}\right)$ and (iv) an approximate frequency of the chamber surface oscillations around the equalization level during the overtravel phenomenon $\Omega\left(=\omega / \omega_{0}\right)$. Note that due to the non-linear damping in the system, the frequency content of those oscillations will change in time. At the onset of the overtravel phenomenon, the basic frequency of the oscillations is $\omega=2 \pi / T_{\text {osc }}$, where $T_{\text {osc }}$ is the time interval between the first two up-crossings of the equalization level in the chamber (see Fig. 1). When deriving the expression for $\Omega$ in table 1, the following approximation has been made:

$$
T_{\text {osc }} \approx 4\left(t_{1}-t_{\text {full }}\right), \text { hence } \Omega \approx \frac{\pi / 2}{\left(\tau_{1}-\tau_{\text {full }}\right)}
$$

Note that table 1 also contains formulae (with a Roman numeral II followed by a letter) which are valid for the special case when the surface area of the upper reservoir is infinitely large (in comparison to the lock chamber surface) and which are retrieved by simply 
putting $\sigma=0$ in the general formulae. As mentioned before, this special case is typically associated to navigation locks being filled from a river or channel reach (with a large forebay).

Formula (IIb) of the present paper (table 1), regarding the filling time, has been presented earlier as formula (5-5) in USACE (2006) and goes back to the formula derived by Pillsbury (1915). Similarly, formula (IIc) for the water level at the first overtravel peak in table 1 has been presented (without explicit derivation) earlier as formula (5-20) in USACE (2006). Formulae similar to (IIb) and (IIc) have also been derived in Dehousse (1985). From the foregoing, it follows that the earlier presented formulae are only valid for the special case of an infinitely large surface of the upper reservoir, though this was not clearly stated in the cited references. The present paper offers formulae, like e.g. (Ib) and (Ic), which have a broader range of applicability than the formulae presented earlier in literature. For the sake of completeness, however, it should be mentioned that Pillsbury (1915) briefly points out how his lock filling time formula should be modified in case of locks in flight, but without providing a complete derivation.

Note that the formulae in table 1 were derived based on the assumption that the culvert valves open instantaneously, i.e. assuming a zero value for the valve opening time $t_{v}$. In practice, this is not feasible (since it would imply an infinite lift force) nor desirable (since it would lead to large surge waves in the lock chamber). In case of a finite opening time of the valves, the lock chamber will fill more slowly. Therefore, it is expected that the formulae (Ic) or (IIc) for the height of the first overtravel peak will lead to overestimates. Regarding the filling time, it is proposed in USACE (2006) to add a term $K t_{v}$, where $K$ is an overall valve coefficient (in the range 0.4 to 0.6 ), to the analytical formula for the filling time $t_{\text {full }}$ in case of instantaneous opening of the culvert valves. In the dimensionless formulation of the present paper, this would lead to adding a term $K \omega_{0} t_{v}$ to the right hand side of formulae (Ib) and (IIb) in table 1.

\subsection{Analytical formulae derived from exact solution of simplified equation}

In this section, some additional formulae will be presented in order to gain some more (physical) insight and for comparison purposes (see sections 2.4 and 4.5) with the formulae derived in section 2.2 .

A first simplification of the governing equation that leads to an exact solution, consists in neglecting damping but still accounting for inertia, i.e. $\psi=0$. Then, Eq. (8) reduces to a linear second-order differential equation, $(1+\sigma) \zeta+\ddot{\zeta}=0$, which combined with the initial conditions in Eq. (10) leads to the solution:

$$
\zeta=-\cos \left[(1+\sigma)^{\frac{1}{2}} \tau\right]=-\cos \left[(1+\sigma)^{\frac{1}{2}} \omega_{\mathrm{o}} t\right]
$$


The analytical formulae for the filling time and overtravel characteristics that can be derived from Eq. (14) are presented in table 2 (i.e. formulae with a Roman numeral III followed by a letter). From Eq. (14) it can be deduced that the inverse time scale $\omega_{0}$ - by means of which the dimensionless time coordinate $\tau$ is defined (Eq. (6)) - corresponds to the frequency of the chamber surface oscillations around the equalization level in case of an undamped system with an infinitely large surface area of the upper reservoir, i.e. if $\psi=\sigma=0$.

A second simplification of the governing equation that is considered, consists in neglecting inertia but still accounting for damping. When inertia is neglected, the $\ddot{\zeta}$ term disappears from Eq. (8), which in combination with the initial conditions of Eq. (10) yields an exact solution:

$$
\zeta=\left[1-\frac{1}{2}\left(\frac{1+\sigma}{\psi}\right)^{\frac{1}{2}} \tau\right]^{2}
$$

The analytical formula for the filling time that can be derived from Eq. (15) is presented as formula (IVb) in table 2. Since no overtravel takes place in an inertialess configuration, table 2 contains no formulae for the overtravel characteristics. Note that for the special case of an inertialess system with an infinitely large surface area of the upper reservoir, i.e. if $\sigma=0$, formula (IVb) in table 2 reduces to:

$$
\tau_{\text {full }}=2 \psi^{\frac{1}{2}}
$$

Converting Eq. (16) for the dimensionless filling time $\tau_{\text {full }}\left(=\omega_{0} t_{f u l l}\right)$ to physical dimensions, by substituting the expressions for $\omega_{\mathrm{o}}=\left(\frac{g n A}{L S_{c}}\right)^{\frac{1}{2}}$ and $\psi=\frac{k}{2} \frac{\ell}{L} \frac{S_{c}}{n A}$, yields:

$$
t_{f u l l}=\frac{2 S_{c} \sqrt{\ell}}{C_{L} n A \sqrt{2 g}}
$$

where the overall lock coefficient $C_{L}$ is defined as in USACE (2006), $C_{L}=1 / \sqrt{k}$.

Equation (17) is a classical formula in textbooks on navigation locks (like e.g. Dehnert (1954)), since it is straightforward to derive for a simple through-the-gate fillingemptying system based upon valves in the lock gates. The latter system can be interpreted as an inertialess limit case of a culvert-based system, i.e. a system in which the culvert length $L \rightarrow 0$. Strictly speaking, this limit case would result in both $\omega_{0} \rightarrow \infty$ and $\psi \rightarrow \infty$. Since the culvert length has no physical meaning any more for a through-the-gate system, any non-zero length scale $L$ can be used to define both $\omega_{\mathrm{o}}$ and $\psi$. Note that the choice of the length scale is not relevant, since the $L$ appearing in the expression for $\omega_{\mathrm{o}}$ and the one appearing in the expression for $\psi$ cancel against each other, yielding formula (17) which is independent of $L$.

Note that Eq. (17), which is derived from Eq. (16) and is valid for an inertialess system (with $\sigma=0$ ), states that the lock filling time is inversely proportional to the number $n$ of identical culverts. This implies that doubling the number of culverts (i.e. filling openings in 
the gates) leads to cutting the lock filling time in half. This will no longer be the case for a system with inertia (with $\sigma=0$ ), as is obvious when formula (IIb) is rewritten as $\tau_{\text {full }}=2^{1 / 2}\left[(1+2 \psi)^{1 / 2}-1\right]$ and is compared to Eq. (16).

\subsection{Numerical validation of analytical formulae}

To assess the error made by neglecting the exponential term in Eq. (11), the approximate analytical formulae in table 1 will be compared to the results of an accurate numerical solution of the original differential Eq. (8) for different values of the parameters $\sigma$ and $\psi$ (see below).

For the parameter $\sigma$, three representative values will be considered $(\sigma=0, \sigma=0.5$ and $\sigma=1)$. For the parameter $\psi$, on the other hand, it is not yet clear what is a relevant range. Ideally, this range should be determined by carefully analysing a large set of navigation locks with different types of culvert-based filling-emptying systems (for which a sufficient amount of data are available).

In table 3, a rough estimate of the range for $\psi$ is made for two types of culvert-based lock filling-emptying systems, i.e. a system with bypass culverts in the lock heads and a sidewall filling system with longitudinal culverts and ports. Firstly, a typical range is estimated for the parameters of which $\psi$ is a function, based both on experience and on references cited hereafter: PIANC (1986) for the lift $\ell$; Dehousse (1985) for the ratio $S_{c} / n A$; Dehnert (1954) for the overall lock coefficient $C_{L}$, which can be translated into a total head loss coefficient $k \approx 1 / C_{L}^{2}$, following USACE (2006). Secondly, the estimated bounds are combined straightforwardly (i.e. making abstraction of the fact that some combinations of bounds might be less realistic) in order to determine a minimum and maximum value for $\psi$. As shown in table 3 , the range $10^{-1} \leq \psi \leq 10^{3}$ is retrieved for carrying out the numerical validation. From the foregoing, it is obvious that the lower and upper bounds that are mentioned in table 3 are only indicative. Although the estimation intentionally gives a wide range, it cannot be excluded that some existing locks are characterized by parameter values falling outside the indicated ranges.

With the aforementioned representative values for the parameters $\sigma$ and $\psi$, accurate numerical solutions of Eqs. (8) and (10) are calculated, based on a fourth-order Runge-Kutta finite difference method (see e.g. Press et al. 1989) with an equidistant dimensionless time step of $10^{-3}$.

The numerically predicted filling time, $\tau_{f u l l}$, is compared to the analytical formula (Ib) in Fig. 3a and 3b. While Fig. 3a shows a good agreement for large values of the parameter $\psi$, the zoom of Fig. $3 b$ reveals that the deviation between the analytical formula and the 
numerical solution increases when $\psi$ approaches zero. This is related to the fact that formula (Ib) erroneously predicts a zero filling time when $\psi=0$, whereas the numerical solutions correctly predict the non-zero filling time which corresponds to the exact solution for the special case of an undamped system, i.e. formula (IIIb). Accepting an arbitrary maximum error of 5\% with respect to the accurate numerical solution, it follows from Fig. $3 \mathrm{~b}$ that formula (Ib) is valid for $\psi \gtrsim 9$.

In Fig. 3c, a comparison is made between the numerically predicted water surface deviation from the equalization level at the first overtravel peak, $\zeta_{1}$, and the analytical formula (Ic). Again, a good agreement is found for large values of the parameter $\psi$, but the agreement deteriorates when $\psi$ approaches zero. While the analytical formula erroneously predicts an infinite limit value for $\zeta_{1}$ when $\psi \rightarrow 0$, the numerical solution yields the correct limit value of $\zeta_{1}=1$, which corresponds to the exact solution for the special case of an undamped system, i.e. formula (IIIc). When adopting the same arbitrary maximum error of $5 \%$ as specified above, it follows from Fig. 3c that formula (Ic) is valid for $\psi \gtrsim 2$.

The numerically predicted frequency of the chamber surface oscillations around the equalization level, $\Omega$, is compared to formula (Ie) in Fig. 3d. Once more, a good agreement is found for large values of the parameter $\psi$, but the agreement deteriorates when $\psi$ approaches zero. For $\psi \rightarrow 0$, the numerical solution yields the correct limit value of $\Omega=(1+\sigma)^{\frac{1}{2}}$, which corresponds to the exact solution for the special case of an undamped system, i.e. formula (III)). The analytical formulae erroneously predict a value which is a factor $\frac{\pi \sqrt{2}}{4}(\approx 1.11)$ too high. Using the same maximum error of $5 \%$ as above, it follows from Fig. $3 \mathrm{~d}$ that formula (Ie) is valid for $\psi \gtrsim 2$.

When comparing the aforementioned validity ranges ( $\psi \gtrsim 9$ resp. $\psi \gtrsim 2)$ to the range $\left(10^{-1} \lesssim \psi \lesssim 10^{3}\right)$ that was conservatively estimated in table 3 , it can be concluded that the analytical formulae are likely to be of use for many practical filling-emptying systems.

Moreover, the numerical validation in this section has demonstrated that neglecting the exponential term in Eq. (11) in the derivation of the analytical formulae (Ia) to (Ie), which was already conjectured by Pillsbury (1915), is acceptable, at least within the aforementioned validity ranges of the analytical formulae.

\subsection{Discussion of analytical formulae}

Figure $3 \mathrm{~b}$ offers a comparison of 4 curves based upon alternative analytical formulae for the filling time $\tau_{f u l l}$ : (i) formula (Ib) for the case of $\sigma=1$, (ii) formula (Ib) for the case of $\sigma=0.5$, (iii) formula (Ib) for the case of $\sigma=0$, which corresponds to formula (IIb), which in turn corresponds to the Pillsbury (1915) formula appearing in USACE (2006) and (iv) formula 
(16), which corresponds to formula (IVb) for the special case of an inertialess configuration with $\sigma=0$.

Note that the predicted filling times of the four curves differ significantly, hence care should be exercised when selecting an appropriate analytical formula for the filling time in a given navigation lock configuration.

For a configuration with finite surface area for the upper reservoir, i.e. $\sigma \neq 0$, it is worth using formula (Ib) derived in this paper instead of the Pillsbury formula in USACE (2006), as can be deduced from Fig. 3b. The Pillsbury formula is only appropriate for a configuration with $\sigma=0$.

Formula (16) - of which the dimensional form (17) is ubiquitous in textbooks and is only valid for an inertialess configuration, like e.g. a through-the-gate system, in case $\sigma=0$ results in a significant overprediction of the filling time for culvert-based systems. Hence, formula (16) is in general not advocated for the latter type of filling-emptying systems, though some practitioners do use it because of its simplicity. Yet, it is worth pointing out that in case of $\sigma=0$, formula (Ib) can be written as $\tau_{f u l l}=2^{1 / 2}\left[(1+2 \psi)^{1 / 2}-1\right]$, from which it is obvious that for large $\psi$ values, the predictions of formula (Ib) approach the ones of formula (16). The latter can indeed be observed in Fig. 3a.

With regard to the first overtravel peak relative to the equalization level, $\zeta_{1}$, Fig. 3c only shows one curve which is based on an analytical formula, i.e. formula (Ic). Note that this formula, which is identical to a formula appearing in USACE (2006), is independent of the parameter $\sigma$.

As far as the dimensionless frequency $\Omega$ of the chamber surface oscillations around the equalization level is concerned, it is worth mentioning that most references in literature, like e.g. USACE (2006), contain no explicit analytical formula for this quantity. Figure $3 \mathrm{~d}$ offers a comparison of 3 curves based upon a single analytical formula that was derived in this paper, i.e. formula (Ie). The three curves correspond to different values for $\sigma$ : (i) $\sigma=1$, (ii) $\sigma=0.5$ and (iii) $\sigma=0$. Similar to the situation with respect to $\tau_{f u l l}$, it is worth taking the dimensions of the upper reservoir into account when predicting $\Omega$ (Fig. 3d).

\section{Extension to more complex culvert geometries: equivalent culvert approach}

While the aforementioned formulae were derived for the case of $n$ identical culverts with constant cross section, culvert-based lock filling-emptying systems often have a more complex geometry. Some examples are: (i) culverts with cross-sectional changes, (ii) nonidentical bypass culverts in lock heads with rolling or sliding gates and (iii) systems of (longitudinal) culverts with ports. Each of these systems can be modelled in good approximation as a hydraulic network of pipes with constant cross section. As will be 
explained below, every two pipes in the network that are connected in series can be substituted by one equivalent culvert. The same goes for every two pipes in the network that are connected in parallel. By repetitively applying these substitutions, any of the aforementioned culvert-based systems can be ultimately modelled by means of one $(n=1)$ equivalent culvert with constant cross section. This will allow application of the analytical formulae derived earlier for the case of $n$ identical culverts with constant cross section.

To substitute two culverts (denoted 1 and 2 ) in series by one equivalent culvert having a constant cross-sectional area $A_{e q}$, a length $L_{e q}$ and a total head loss coefficient $k_{e q}$, the following equivalency relations should be applied:

$$
\begin{aligned}
& \frac{k_{e q}}{A_{e q}^{2}}=\frac{k_{1}}{A_{1}^{2}}+\frac{k_{2}}{A_{2}^{2}} \\
& \frac{L_{e q}}{A_{e q}}=\frac{L_{1}}{A_{1}}+\frac{L_{2}}{A_{2}}
\end{aligned}
$$

These relations follow straightforwardly from writing a combined Bernoulli equation for both culverts in series and for the equivalent culvert:

$$
\Delta z=\frac{k_{1}}{2 g A_{1}^{2}}|Q| Q+\frac{L_{1}}{g A_{1}} Q^{\prime}+\frac{k_{2}}{2 g A_{2}^{2}}|Q| Q+\frac{L_{2}}{g A_{2}} Q^{\prime}=\frac{k_{\mathrm{eq}}}{2 g A_{e q}^{2}}|Q| Q+\frac{L_{e q}}{g A_{e q}} Q^{\prime}
$$

where $\Delta z$ denotes the total difference in head over the two culverts in series.

In a similar way, one can attempt to derive equivalency relations for two culverts in parallel by writing a Bernoulli equation for each culvert and for the equivalent culvert:

$$
\begin{aligned}
\Delta z & =\frac{k_{1}}{2 g A_{1}^{2}}\left|Q_{1}\right| Q_{1}+\frac{L_{1}}{g A_{1}} Q_{1}^{\prime}=\frac{k_{2}}{2 g A_{2}^{2}}\left|Q_{2}\right| Q_{2}+\frac{L_{2}}{g A_{2}} Q_{2}^{\prime} \\
& =\frac{k_{\mathrm{eq}}}{2 g A_{e q}^{2}}\left|Q_{1}+Q_{2}\right|\left(Q_{1}+Q_{2}\right)+\frac{L_{\mathrm{eq}}}{g A_{e q}}\left(Q_{1}^{\prime}+Q_{2}^{\prime}\right)
\end{aligned}
$$

where $\Delta z$ denotes the difference in head over each of the two culverts in parallel.

No straightforward equivalency relations can be derived from Eq. (21), unless some extra condition is imposed. E.g. assuming all head loss terms in Eq. (21) to be equal, yields:

$$
\frac{A_{e q}}{k_{e q}{ }^{1 / 2}}=\frac{A_{1}}{k_{1}^{1 / 2}}+\frac{A_{2}}{k_{2}^{1 / 2}}
$$

Consequently, all inertia terms should then be equal too, which leads to:

$$
\frac{A_{e q}}{L_{e q}}=\frac{A_{1}}{L_{1}}+\frac{A_{2}}{L_{2}}
$$

Note that Eqs. similar to (18), (19), (22) and (23) have been proposed earlier by Escande $(1942,1943)$ in the field of surge tank design for a hydroelectric power plant with complex feeder pipe lay-outs. 
Since there are two equivalency relations defining the three parameters of the equivalent culvert, one parameter can be chosen freely, e.g. $A_{e q}$.

With the equivalent culvert approach, a complex lock filling-emptying system can ultimately be replaced by one single equivalent culvert. The corresponding governing equation for the dimensionless water level in the lock chamber $\zeta$, function of the dimensionless time $\tau$, can then be written as follows:

$$
(1+\sigma) \zeta+\psi_{e q}|\dot{\zeta}| \dot{\zeta}+\ddot{\zeta}=0
$$

where

$$
\psi_{e q}=\frac{k_{e q}}{2} \frac{\ell}{L_{e q}} \frac{S_{c}}{n_{e q} A_{e q}}
$$

and

$$
\tau=\omega_{0, e q} t=\left(\frac{g n_{e q} A_{e q}}{L_{e q} S_{c}}\right)^{\frac{1}{2}} t
$$

with $n_{e q}=1$.

As a consequence, the analytical formulae in table 1 and their range of validity remain applicable upon substituting $\psi$ by $\psi_{e q}$.

Note that for predicting lock characteristics other than those mentioned in table 1 - for example, the distribution of the filling (or emptying) discharges and the associated surge waves in the lock chamber - replacement of a (complex) filling-emptying system with one equivalent culvert may not always be an adequate modelling strategy.

\section{Experimental validation of analytical formulae}

To assess the validity of the approximate analytical solutions of the complete equation, an experimental validation is carried out. The experimental set-up and methodology will be explained first. Then the application of the analytical formulae will be discussed.

Additionally, some numerical simulation work with a hydraulic network model will be introduced. Finally, the analytical and numerical predictions will be compared with the experimental results.

\subsection{Experimental set-up and methodology}

Figure 4 presents a schematic plan view of the experimental set-up, with the most important dimensions. More details can be found in Schindfessel (2013). Two prismatic reservoirs, i.e. the upper reservoir (with a surface area $S_{u}=7.53 \mathrm{~m}^{2}$ ) and the lock chamber (with a surface area $S_{c}=2.45 \mathrm{~m}^{2}$ ), are connected by culverts. Each culvert is sealed by a vertical lift valve. All valves are opened manually in a nearly synchronous and instantaneous way. 
In a first series of lock filling tests, both reservoirs merely act as communicating vessels. These tests will be referred to as experiments with a finite upper reservoir, characterized by a value of $\sigma=S_{c} / S_{u}=0.325$. In a second series of tests, it is attempted to keep the water level in the upper reservoir $z_{u}$ constant in time, by constantly adding discharge to the upper reservoir while allowing the excess discharge to be drained over a spillway construction. Such a configuration corresponds to the case of an infinitely large surface area of the upper reservoir, hence $\sigma=0$. Due to the limited size of the spillway, however, still a (limited) variation in time of $z_{u}$ is observed in these tests. Therefore, the second series of tests will be referred to as tests with a quasi infinite upper reservoir, denoted as $\sigma \approx 0$.

In both series of tests, two distinct types of lock filling systems will be implemented. The first type concerns systems which consist of $n(=1$ or 2$)$ identical culverts in parallel, allowing a direct comparison of the experimental results with the analytical formulae derived in section 2.2. The second type consists of systems which require the equivalent culvert approach of section 3 to be applied, before a comparison of analytical results and measurements is feasible.

For the first type of systems, culverts with three different lengths will be considered. In table 4 (and subsequent tables), each of those systems will get a unique identifier consisting of the number of identical culverts (i.e. $n$ ) followed by two characters corresponding to the length of the culvert: short culvert (SC), medium length culvert (MC) and long culvert (LC).

For the second type of systems, either systems composed of two bypass culverts with different lengths (e.g. 1SC+1MC) will be considered or systems consisting of one longitudinal culvert with ports (e.g. $1 \mathrm{MC}$ with 2 ports). Note in table 4 that, though not strictly necessary, also system $2 \mathrm{MC}$ will be modelled with the equivalent culvert approach, for comparison purposes with the results based upon the $n$ identical culverts model.

All culverts in the aforementioned lock filling systems are implemented in the model in a modular way by means of plastic (polyvinyl chloride, PVC) components (i.e. straight pipes, bends, T-junctions, etc.) having a constant diameter $(D=0.1036 \mathrm{~m})$, hence a constant cross-sectional area $\left(A=8.4310^{-3} \mathrm{~m}^{2}\right)$.

An overview of the parameters characterizing the different lock-filling systems is given in table 4. The $k$ values in the table will be discussed in section 4.2. Note that different values for the lift have been used for the case of a quasi infinite upper reservoir $(\ell \approx 0.275 \mathrm{~m}$, with one exception, i.e. the system referred to as " $1 \mathrm{LC}$ reduced lift", for which $\ell \approx 0.138 \mathrm{~m}$ ) and for the finite upper reservoir case $(\ell \approx 0.185 \mathrm{~m}$, except for $1 \mathrm{LC}$ reduced lift, for which $\ell \approx 0.082 \mathrm{~m})$. 
During the lock filling tests, water heights in the upper reservoir and in the lock chamber are measured with two Cerabar S pressure sensors, having a measurement accuracy of $0.5 \mathrm{~mm}$ according to the technical specifications. Both sensors are connected to an Arduino micro-controller board, which is logging at a rate of $10 \mathrm{~Hz}$. The measurement location of the pressure sensor in the upper reservoir is fixed, whereas the sensor in the lock chamber can be mounted in four alternative locations, as is schematically indicated in Fig. 4. The measurement devices are calibrated, and each time they are moved, a reference water height is read by means of a point gauge.

A given lock filling test is repeated several times, such that at least two measurements are carried out in each of the alternative locations, to ensure that the average lock filling curve (i.e. the arithmetic average of the individual, validated lock filling curves) is representative. From the average filling curve, the lock filling time $t_{f u l}$, the height of the first overtravel peak $\left(z_{1}-z_{e}\right)$ and the frequency of the chamber surface oscillations around the equalization level $\omega$ are derived and reported in tables 5,6 and 7, respectively.

The water level measurements are judged to be reproducible, since the individual lock filling curves agree well. Moreover, it can be deduced from Fig. 5 that the individual heights of the first overtravel peak $\left(z_{1}-z_{e}\right)$ only show a limited scatter around the reported one (i.e. the value derived from the average lock filling curve) of about $\pm 1 \mathrm{~mm}$.

Besides the unsteady lock filling tests, also some steady state experiments have been run, in order to empirically derive the head loss characteristics of the distinct culverts. Those runs are characterized by constant water levels in both the upper reservoir and the lock chamber. The latter requires the water entering the lock chamber through the culverts to be evacuated via an extra opening in the downstream wall of the lock chamber, sealed by a valve (see Fig. 4).

\subsection{Analytical predictions}

The analytical formulae from table 1 require the parameter $\psi$ (or $\psi_{e q}$ ) to be known, which in turn requires estimates to be available for the total head loss coefficient $k$ of the distinct culverts.

Though $k$ is meant to be a global coefficient for a lock filling system, it was determined by modelling a given culvert first as a hydraulic network consisting of pipes (i.e. frictional head losses) and resistances (i.e. minor head losses). Such an approach is also advocated in USACE (2006).

To set up and numerically simulate the hydraulic network models, the LOCKSIM software package developed by Schohl (1998) is used. LOCKSIM uses an explicit approximation to the Colebrook formula in order to calculate the Darcy-Weisbach friction 
coefficient $\lambda$ for turbulent pipe flow as a function of the relative wall roughness and the Reynolds number. When the Reynolds number drops below a user-specified threshold value (default value $=2000$ ) LOCKSIM switches to the classical $\lambda$ formula for laminar pipe flow . For the minor head loss coefficients of the resistances, constant values specified by the user are adopted in this work.

Firstly, numerical hind casts of the steady-state experiments are made, in order to obtain an estimate of the relative wall roughness and the minor head losses for the distinct culverts. Secondly, the relative wall roughness is further optimized by hind casting some unsteady lock filling tests. Since the flow in the culverts is not fully turbulent throughout the lock filling and overtravel process, there is a Reynolds number dependency, hence a time dependency of the friction coefficient. Yet, by carefully examining the corresponding time series of the calculated friction coefficient, it turns out that for each culvert (with length $L$ and diameter $D$ ) an average friction coefficient $\lambda_{\text {avg }}$ can be defined, which is representative for a large part of the filling process (but not for the overtravel process, where large peaks in $\lambda$ are observed around the crests and troughs of the water level oscillations). Finally, the total head loss coefficient of the culvert $k$ is then obtained by adding a contribution $\lambda_{\text {avg }} L / D$ to the earlier estimated minor head loss coefficients of the other resistances in the culvert. The $k$ values specified in table 4 are obtained by the aforementioned procedure, allowing the calculation of the corresponding parameters $\psi\left(\right.$ or $\psi_{e q}$ ). In order to calculate $\psi_{e q}, A_{e q}$ is chosen equal to $A$, as suggested in section 3 .

The parameter $\psi\left(\right.$ or $\left.\psi_{e q}\right)$ for the different lock filling systems used in the experimental validation ranges from 5.8 to 24.9 (table 4). These values are (to a large extent) situated in the ranges ( $\psi \gtrsim 9$ resp. $\psi \gtrsim 2$, see table 1$)$ where the analytical formulae are assumed to be valid, but are still small enough that differences between the governing equation and the approximate analytical formulae are measurable.

From table 4 it follows that the $\psi\left(\right.$ or $\psi_{e q}$ ) parameters corresponding to the $2 \mathrm{MC}$ system when modelled by means of an equivalent culvert approach do not differ from the values obtained when adopting the $n(=2)$ identical culverts model. Consequently, the corresponding lock filling and overtravel characteristics predicted by the analytical formulae will not differ either.

The predictions of $t_{f u l l},\left(z_{1}-z_{e}\right)$ and $\omega$ based on the analytical formulae, are presented in tables 5,6 and 7, respectively, for all considered lock filling systems. With respect to table 5 , it is worth noting that formulae (Ib) and (IIb) have been applied to all systems, i.e. even for those which are characterized by a $\psi\left(\right.$ or $\psi_{e q}$ ) parameter outside the validity range $\psi \gtrsim 9$. In tables 6 and 7, formulae (Ic) and (Ie), respectively, have been applied within their range of validity $\psi \gtrsim 2$. 
The analytical results will be further discussed in section 4.5.

\subsection{Numerical predictions}

To facilitate the interpretation of differences between analytically predicted and measured quantities for the case $\sigma \approx 0$ in section 4.5 , some numerical predictions with the LOCKSIM software are included in tables 5,6 and 7. Distinction is made between three types of LOCKSIM models.

In the first type of model, referred to as LOCKSIM1, it is assumed - analogous to the analytical formulae - that both the water level in the upper reservoir and the head losses are constant in time. The latter implies that the Reynolds number dependency of the friction coefficient is neglected.

The second type of model, referred to as LOCKSIM2, only differs from LOCKSIM1 in that the variation in time of the water level in the upper reservoir (due to the limited dimensions of the spillway in the $\sigma \approx 0$ configuration of the experimental set-up) is accounted for. The magnitude of these variations depends on the specific lock-filling system, but is observed to be at most $12 \mathrm{~mm}$ for the tests with a lift of about $275 \mathrm{~mm}$.

Finally, the third type of model, referred to as LOCKSIM3, differs from LOCKSIM2 in that the Reynolds number dependency of the friction coefficient is taken into account.

For the lock filling systems which consist of $n$ identical culverts, the hydraulic network in the LOCKSIM simulations will include all culverts, whereas for the lock filling systems which are to be modelled analytically with the equivalent culvert approach, the hydraulic network in the LOCKSIM simulations will consist of one single culvert, i.e. the equivalent culvert with known characteristics $\left(A_{e q}, L_{e q}, k_{e q}\right)$. As a consequence, it is not possible to account for the Reynolds dependency of the friction coefficient in case of the equivalent culvert, hence no LOCKSIM3 results will be reported for the corresponding lock filling systems in tables 5 and 6.

\subsection{Results}

In tables 5, 6 and 7 the analytical and numerical predictions are compared to the experimental values for $t_{f u l l},\left(z_{1}-z_{e}\right)$ and $\omega$, respectively.

\subsection{Discussion}

From table 5, it follows that formula (Ib) somewhat underestimates the measured lock filling time $t_{\text {full }}$. The deviations are slightly smaller for the $\sigma=0.325$ case (making abstraction of the "1LC reduced lift" test, which has a $\psi$ parameter clearly out of the validity range for formula 
(Ib)) as compared to the $\sigma \approx 0$ case. For the latter case, the results of the various LOCKSIM models (and in particular the clear improvement of LOCKSIM2 predictions in comparison to LOCKSIM1) suggest that these deviations are mainly due to formula (Ib) not accounting for water level fluctuations in the upper reservoir (which are indeed observed during the experimental tests with $\sigma \approx 0$ ) and virtually not to neglecting the Reynolds number dependency of the head losses (since LOCKSIM3 results are nearly identical to LOCKSIM2).

Note in table 5, that the predictions of formula ( $\mathrm{Ib}$ ) show the same behaviour as the measurements with respect to doubling of the number of culverts, i.e. the filling time is reduced by more than $50 \%$.

For comparison purposes, predictions of the lock filling time based upon formulae (IIb) and (16) are also shown in table 5. Note that formula (IIb), which appears in USACE (2006), gives identical results as formula (Ib) for the case of $\sigma \approx 0$, but leads to overestimates of the filling time for the $\sigma=0.325$ case. These deviations would even be larger in case of higher values for $\sigma$. Formula (16), which is a classical textbook formula that is easily derived for filling through openings in the lock gates, significantly overestimates the lock filling time, which is due to the fact that inertia is not accounted for in formula (16). Moreover, formula (16) assumes an infinitely large surface area of the upper reservoir, which explains why the overestimates are larger for the finite upper reservoir case as compared to the quasi infinite case. The LOCKSIM calculations indicate that if experiments would be performed with a truly infinitely large upper reservoir, the measured filling time would decrease. Thus, the performance of formula (Ib) would be better, while formula (16) would show an increased error.

From table 6, it can be concluded that formula (Ic), which is identical to the formula appearing in USACE (2006), overestimates the height of the first overtravel peak. The deviations are (with few exceptions) higher than the accuracy of the reported measurements that was estimated in Fig. 5 to be about $\pm 1 \mathrm{~mm}$. The deviations are smaller for the $\sigma=0.325$ case as compared to the $\sigma \approx 0$ case. For the latter case, the results of the various LOCKSIM models (and in particular the clear improvement of LOCKSIM2 predictions in comparison to LOCKSIM1 results) suggest that this is to a large extent due to formula (Ic) not accounting for the observed water level fluctuations in the upper reservoir. Consider for example the experiment $2 \mathrm{MC}$ of the $\sigma \approx 0$ case, where the deviation between prediction and experiment is the largest. Comparing the LOCKSIM1 with the LOCKSIM2 case shows that the influence of the water level variation in the upper reach is substantial. Indeed, this experiment featured the largest water level variations in the upper reach.

Contrary to what was observed for the lock filling time predictions of formula (Ib), however, the fact that the Reynolds number dependency of the head losses is neglected also 
clearly worsens the predictions of formula (Ic) for the height of the first overtravel peak (as can be deduced from a comparison of the LOCKSIM3 and LOCKSIM2 predictions). The latter observation seems logical since the predictions of formula (Ic) are based on a constant total head loss coefficient, i.e. on a constant average friction factor $\lambda_{\text {avg }}$, whereas especially during the overtravel, the values of $\lambda$ are on average somewhat larger and are characterized by high peaks when crests or troughs of the water level oscillations are reached. Moreover, in section 4.2 it was also explained that the average friction factor $\lambda_{\text {avg }}$ was representative for filling prior to the overtravel. As a consequence, the adopted procedure to determine the $k$ values, hence the values of $\psi\left(\right.$ or $\left.\psi_{e q}\right)$, tends to favour the predictions of the filling time according to formula (Ib) and penalize the predictions of the height of the first overtravel peak based upon formula (Ic). Since the Reynolds numbers are higher for a prototype than in a corresponding scale model, $k$ is expected to vary less during filling, leading to an improved prediction of the filling time. It is not clear whether this also holds for the prediction of the first overtravel peak, since even for a prototype, the Reynolds number varies considerably during overtravel.

Note in table 6, that the predictions of formula (Ic) show the same behaviour as the measurements with respect to doubling of the number of culverts, i.e. the height of the first overtravel peak is quasi doubled. Moreover, both formula (Ic) and the measurements yield a height of the first overtravel peak that is independent of the lift (compare results of 1LC and 1LC reduced lift).

From table 7, it follows that formula (Ie) might lead to either over- or underestimation of the frequency $\omega$ of the chamber surface oscillations around the equalization level. Both the predictions of formula (Ie) and the measurements show that the frequency $\omega$ is (quasi) independent of the lift. With respect to doubling of the number of culverts, formula (Ie) predicts that the frequency increases with a factor $(\sqrt{2} \approx) 1.41$, while the measurements show a factor ranging from 1.31 to 1.64 . Note that $\omega$ was calculated by means of Eq. (13). This and the possible presence of non-linear damping, can explain why formula (Ie) shows both overand underestimation.

Summarizing the abovementioned observations, the following general conclusions can be drawn from tables 5, 6 and 7. The analytical formulae (Ib), (Ic) and (Ie) give a reasonable agreement with the measurements for systems consisting of $n$ identical culverts. In combination with the equivalent culvert approach, the same quality of predictions is achieved for the considered systems with non-identical bypass culverts or with longitudinal culverts and ports. 


\section{Conclusions}

The analytical formulae designated as Ia, Ib, Ic, Id, and Ie in table 1 for lock filling (or emptying) and overtravel characteristics of culvert-based systems are valid for an arbitrary ratio $\sigma$ of lock chamber surface area to upper (resp. lower) reservoir surface area, meaning that they are valid for locks with constant upper (resp. lower) reservoir levels as well as for configurations like e.g. staircase locks and double-lift locks. The importance of taking into account both the specific $\sigma$ ratio of a lock configuration and the inertia in the culvert flow is illustrated by the numerical and the experimental results.

The analytical formulae have been derived for culvert-based systems consisting of $n$ identical culverts but more complex culvert layouts can be modelled by using an equivalent culvert approach.

By means of an accurate numerical solution of the governing non-linear second-order differential equation, it is possible to present validity ranges of the analytical formulae, in terms of a non-dimensional damping parameter $\psi$. Based on an estimate of minimal and maximal parameter values for two types of culvert-based lock filling (and emptying) systems, it can be concluded that the analytical formulae are applicable to many lock configurations. Within the demonstrated validity ranges, the analytical formulae can be used as a design tool along with numerical and physical modelling, especially in the early stages of navigation lock design. Additionally, the numerical validation implicitly demonstrates the conjecture of Pillsbury (1915) - i.e. that an exponential term in the first integration of the governing differential equation can be neglected to good approximation. Moreover, the (dimensionless) analytical formulations provide an elegant framework for comparing the characteristics of existing locks.

For the considered lay-outs of filling (and emptying) systems, the agreement between the formulae and the experimentally measured lock filling and overtravel characteristics is satisfactory. Additional testing is needed to verify whether this agreement also extends to other, more complex filling (and emptying) systems.

\section{Acknowledgements}

The authors are grateful to the technical staff of the Hydraulics Laboratory for their contribution to the experimental set-up and acknowledge Dr. G.P. Schramkowski of Flanders Hydraulics Research for stimulating discussions, as well as the anonymous reviewers and the associated editor for their valuable comments. The first author is Ph.D. fellow of the Research Foundation - Flanders (FWO) and the third author is Ph.D. fellow of the Special Research Fund (BOF) of Ghent University. 


\section{Notation}

$A, A_{i}, A_{e q}=$ cross-sectional area of a(n) (equivalent) culvert ( $\left.\mathrm{m}^{2}\right)$

$C_{L}=$ overall lock coefficient (-)

$D=$ diameter of pipes with circular cross section (m)

$g=$ gravitational acceleration $\left(\mathrm{ms}^{-2}\right)$

$k, k_{i}, k_{e q}=$ total head loss coefficient of a(n) (equivalent) culvert (-)

$K=$ overall valve coefficient (-)

$L, L_{i}, L_{e q}=$ length of a(n) (equivalent) culvert (m)

$\ell=\operatorname{lift}(\mathrm{m})$

$n, n_{e q}=$ (equivalent) number of identical culverts (-)

$Q, Q_{i}=$ flow rate in a culvert $\left(\mathrm{m}^{3} \mathrm{~s}^{-1}\right)$

$S_{c}, S_{u}=$ surface area of lock chamber, resp. upper reservoir $\left(\mathrm{m}^{2}\right)$

$t=$ time with respect to start of lock filling (s)

$t_{1}=$ time of first overtravel peak (s)

$t_{\text {full }}=$ filling time $(\mathrm{s})$

$t_{v}=$ valve opening time $(\mathrm{s})$

$T_{\text {osc }}=$ time interval between first two up-crossings of equalization level in lock chamber (s)

$z=$ water level (with respect to lock chamber floor) (m)

$z_{c}, z_{u}=$ water level in lock chamber, resp. upper reservoir (m)

$z_{e}=$ water level at equalization $(\mathrm{m})$

$z_{\mathrm{o}}=$ initial water level in lock chamber $(\mathrm{m})$

$z_{1}=$ water level in lock chamber at first overtravel peak $(\mathrm{m})$

$\Delta z=$ difference in head $(\mathrm{m})$

$\zeta=$ dimensionless water surface deviation from equalization level in lock chamber (-)

$\zeta_{1}=$ dimensionless water level deviation in lock chamber at first overtravel peak (-)

$\lambda, \lambda_{\text {avg }}=($ average $)$ Darcy-Weisbach friction coefficient (-)

$\sigma=$ ratio of surface areas (-)

$\tau=$ dimensionless time coordinate with respect to start of lock filling (-)

$\tau_{1}=$ dimensionless time of first overtravel peak (-)

$\tau_{\text {full }}=$ dimensionless filling time (-)

$\psi, \psi_{\text {eq }}=$ (equivalent) dimensionless damping parameter (-)

$\omega=$ frequency of chamber surface oscillations around equalization level $\left(\mathrm{s}^{-1}\right)$

$\omega_{0}, \omega_{0, e q}=($ equivalent $)$ inverse time scale $\left(\mathrm{s}^{-1}\right)$

$\Omega=$ dimensionless frequency of chamber surface oscillations around equalization level (-)

' = derivative with respect to $t$ 
$=$ derivative with respect to $\tau$

\section{References}

Dehnert, H. (1954). Schleusen und hebewerke: Handbibliothek für bauingenieure. SpringerVerlag, Berlin.

Dehousse, N. (1985). Les écluses de navigation. Laboratoires d'Hydrodynamique, d'Hydraulique Appliquée et de Constructions Hydrauliques de l'Université de Liège, Liège.

Escande, L. (1942, 1943). Chambre d'équilibre commune à plusieurs canaux d'amenée. C. $R$. Acad. Sci. 215, 245-247, 501-503; 216, 31-33; 144-146.

Jaeger, C. (1956). Mass oscillations in surge systems. In Engineering fluid mechanics. C. Jaeger, ed. Blackie \& Son Limited, London, 189-223.

Permanent International Association of Navigation Congresses (PIANC) (1986). Final report of the international commission for the study of locks. PIANC, Brussels, Belgium.

Pillsbury, G.B. (1915). Excess head in the operation of large locks through the momentum of the column of water in the culverts. Professional memoirs. 7(31), 206-212.

Press, W., Flannery, B., Teukolsky, S., Vetterling, W. (1989) Numerical recipes - The art of scientific computing. Cambridge University Press, New York.

Reisman, A., Silvers, A. (1967). On a non-linear differential equation common to several problems in hydraulics. J. Hydrology. 5, 171-178.

Schindfessel, L. (2013). On inertia phenomena in navigation lock filling and emptying, Master Thesis. Department of Civil Engineering, Ghent University, Belgium.

Schohl, G.A. (1998). User's manual for LOCKSIM: Hydraulic simulation of navigation lock filling and emptying systems. Tennessee Valley Authority, Norris.

Steber, G., Reisman A. (1969). On a non-linear differential equation common to several problems in hydraulics: an addendum. J. Hydrology. 8, 336-340.

U.S. Army Corps of Engineers (USACE) (2006). Hydraulic design of navigation locks. EM 1110-2-1604, US Army Corps of Engineers, Washington DC, USA. 
Table 1 Analytical formulae based on approximate solution of complete equation, including both damping and inertia

\begin{tabular}{|c|c|c|c|c|c|}
\hline Item & $\begin{array}{l}\text { General formulae: } \sigma \neq 0 \\
\text { Finite surface area of upper res }\end{array}$ & rvoir & $\begin{array}{r}\text { Special case: } \sigma=0 \\
\text { Infinite surface area of uppe }\end{array}$ & servoir & $\begin{array}{l}\text { Validity of } \\
\text { formulae }\end{array}$ \\
\hline$\zeta(\tau)$ & $\frac{1}{2 \psi}-\left[\left(\frac{1}{2 \psi}+1\right)^{\frac{1}{2}}-\frac{1}{2}\left(\frac{1+\sigma}{\psi}\right)^{\frac{1}{2}} \tau\right]^{2}$ & (Ia) & $\frac{1}{2 \psi}-\left[\left(\frac{1}{2 \psi}+1\right)^{\frac{1}{2}}-\frac{1}{2}\left(\frac{1}{\psi}\right)^{\frac{1}{2}} \tau\right]^{2}$ & (IIa) & If $\dot{\zeta} \geq 0$, i.e. $0 \leq \tau \leq \tau_{1}$ \\
\hline$\tau_{f u l l}$ & $2\left(\frac{\psi}{1+\sigma}\right)^{\frac{1}{2}}\left[\left(\frac{1}{2 \psi}+1\right)^{\frac{1}{2}}-\left(\frac{1}{2 \psi}\right)^{\frac{1}{2}}\right]$ & (Ib) & $2 \psi^{\frac{1}{2}}\left[\left(\frac{1}{2 \psi}+1\right)^{\frac{1}{2}}-\left(\frac{1}{2 \psi}\right)^{\frac{1}{2}}\right]$ & (IIb) & Error $\lesssim 5 \%$ if $\psi \gtrsim 9$ \\
\hline$\zeta_{1}$ & $\frac{1}{2 \psi}$ & (Ic) & $\frac{1}{2 \psi}$ & (IIc) & Error $\lesssim 5 \%$ if $\psi \gtrsim 2$ \\
\hline$\tau_{1}$ & $\tau_{f u l l}+\left(\frac{2}{1+\sigma}\right)^{\frac{1}{2}}$ & (Id) & $\tau_{f u l l}+\sqrt{2}$ & (IId) & Error $\lesssim 5 \%$ if $\psi \gtrsim 2$ \\
\hline$\Omega$ & $\frac{\pi \sqrt{2}}{4}(1+\sigma)^{\frac{1}{2}}$ & (Ie) & $\frac{\pi \sqrt{2}}{4}$ & (IIe) & Error $\lesssim 5 \%$ if $\psi \gtrsim 2$ \\
\hline
\end{tabular}


Table 2 Analytical formulae based on exact solutions of simplified equations

$$
\text { Simplified equation Simplified equation }
$$

Item without damping and with inertia with damping and without inertia

\begin{tabular}{ccccc}
\hline$\zeta(\tau)$ & $-\cos \left[(1+\sigma)^{\frac{1}{2}} \tau\right]$ & (IIIa) & {$\left[1-\frac{1}{2}\left(\frac{1+\sigma}{\psi}\right)^{\frac{1}{2}} \tau\right]^{2}$} & (IVa) \\
$\tau_{\text {full }}$ & $\frac{\pi}{2}\left(\frac{1}{1+\sigma}\right)^{\frac{1}{2}}$ & (IIIb) & $2\left(\frac{\psi}{1+\sigma}\right)^{\frac{1}{2}}$ & (IVb) \\
$\zeta_{1}$ & 1 & (IIIc) & No overtravel & \\
$\tau_{1}$ & $\tau_{\text {full }}+\frac{\pi}{2}\left(\frac{1}{1+\sigma}\right)^{\frac{1}{2}}$ & (IIId) & No overtravel \\
$\Omega$ & $(1+\sigma)^{\frac{1}{2}}$ & (IIIe) & No overtravel \\
\hline
\end{tabular}

Table 3 Estimation of a range for the parameter $\psi$

\begin{tabular}{ccccc}
\hline & \multicolumn{2}{c}{$\begin{array}{c}\text { Bypass culverts in lock heads } \\
\text { Typical range }\end{array}$} & \multicolumn{2}{c}{$\begin{array}{c}\text { Longitudinal culverts with ports } \\
\text { Typical range }\end{array}$} \\
\hline$\ell(\mathrm{m})$ & 0.2 & 10 & 5 & 15 \\
$L(\mathrm{~m})$ & 270 & 6 & 500 & 50 \\
$\frac{S_{c}}{n A}(-)$ & 100 & 350 & 50 & 200 \\
$C_{L}(-)$ & 0.7 & 0.5 & 0.6 & 0.4 \\
$k \approx \frac{1}{C_{L}^{2}}(-)$ & 2.04 & 4.00 & 2.78 & 6.25 \\
$\psi=\frac{k}{2} \frac{\ell}{L} \frac{S_{c}}{n A}(-)$ & 0.08 & 1167 & 0.7 & 188 \\
\hline
\end{tabular}


Table 4 Overview of parameters characterizing the different lock-filling systems

\begin{tabular}{llcccccc}
\hline & & \multicolumn{6}{c}{ Systems for which “ $n$ identical culverts" model applies } \\
\cline { 3 - 8 } Upper reservoir & Parameter & $1 \mathrm{SC}$ & $1 \mathrm{MC}$ & $2 \mathrm{MC}$ & $1 \mathrm{LC}$ & $2 \mathrm{LC}$ & 1LC reduced lift \\
\hline & $L(\mathrm{~m})$ & 3.78 & 6.58 & 6.58 & 9.48 & 9.48 & 9.48 \\
& $\omega_{\mathrm{o}}\left(\mathrm{s}^{-1}\right)$ & 0.094 & 0.072 & 0.101 & 0.060 & 0.084 & 0.060 \\
& $k(-)$ & 2.36 & 3.81 & 3.81 & 4.59 & 4.59 & 4.59 \\
finite $(\sigma=0.325)$ & $\psi(-)$ & - & 15.5 & 7.8 & 13.1 & 6.6 & 5.8 \\
quasi infinite $(\sigma \approx 0)$ & $\psi(-)$ & 24.9 & 23.3 & 11.7 & 19.4 & 9.8 & 9.7 \\
\hline & & \multicolumn{6}{c}{ Systems to be modelled with equivalent culvert approach } \\
\cline { 3 - 8 } Upper reservoir & Parameter & $2 \mathrm{MC}$ & $1 \mathrm{SC}+1 \mathrm{MC}$ & $1 \mathrm{SC}+1 \mathrm{LC}$ & $1 \mathrm{MC}+1 \mathrm{LC}$ & $1 \mathrm{MC}$ with 2 ports & $1 \mathrm{LC}$ with 2 ports \\
\hline & $L_{e q}(\mathrm{~m})$ & 3.29 & 2.40 & 2.70 & 3.88 & 6.58 & 9.56 \\
& $\omega_{\mathrm{o}, e q}\left(\mathrm{~s}^{-1}\right)$ & 0.101 & 0.119 & 0.112 & 0.093 & 0.072 & 0.059 \\
& $k_{e q}(-)$ & 0.95 & 0.74 & 0.80 & 1.04 & 3.19 & 4.00 \\
finite $(\sigma=0.325)$ & $\psi_{e q}(-)$ & 7.8 & 8.3 & 8.0 & 7.3 & 13.0 & 11.3 \\
quasi infinite $(\sigma \approx 0)$ & $\psi_{e q}(-)$ & 11.7 & 12.3 & 11.8 & 10.8 & 19.4 & 16.8 \\
\hline
\end{tabular}


Table 5 Filling time $t_{\text {full }}$ as predicted by analytical formulae (Ib), (IIb), (18) and LOCKSIM, in comparison to the value derived from the measurements

\begin{tabular}{|c|c|c|c|c|c|c|c|}
\hline \multirow[b]{2}{*}{ Upper reservoir } & \multirow[b]{2}{*}{ Parameter $t_{\text {full }}$} & \multicolumn{6}{|c|}{ Systems for which " $n$ identical culverts" model applies } \\
\hline & & $1 \mathrm{SC}$ & $1 \mathrm{MC}$ & $2 \mathrm{MC}$ & $1 \mathrm{LC}$ & 2LC & 1LC reduced lift \\
\hline \multirow[t]{5}{*}{ finite $(\sigma=0.325)$} & measured (s) & - & 84.0 & 40.0 & 90.0 & 42.2 & 59.2 \\
\hline & predicted (Ib) (s) & - & 79.7 & 37.2 & 86.7 & 40.1 & 52.2 \\
\hline & predicted (Ib)/measured (-) & - & 0.95 & 0.93 & 0.96 & 0.95 & 0.88 \\
\hline & predicted (IIb)/measured (-) & - & 1.09 & 1.07 & 1.11 & 1.09 & 1.02 \\
\hline & predicted (16)/measured (-) & - & 1.31 & 1.38 & 1.35 & 1.44 & 1.36 \\
\hline \multirow[t]{8}{*}{ quasi infinite $(\sigma \approx 0)$} & measured (s) & 100.5 & 124.5 & 61.8 & 133.5 & 64.4 & 91.2 \\
\hline & predicted (Ib or IIb) (s) & 91.8 & 116.4 & 55.0 & 125.9 & 59.1 & 83.4 \\
\hline & predicted (Ib or IIb)/measured (-) & 0.91 & 0.93 & 0.89 & 0.94 & 0.92 & 0.91 \\
\hline & predicted (16)/measured (-) & 1.05 & 1.08 & 1.09 & 1.11 & 1.15 & 1.14 \\
\hline & predicted (LOCKSIM1)/measured (-) & 0.93 & 0.95 & 0.92 & 0.96 & 0.95 & 0.95 \\
\hline & predicted (LOCKSIM2)/measured (-) & 0.96 & 0.98 & 0.98 & 0.98 & 1.01 & 0.98 \\
\hline & predicted (LOCKSIM3)/measured (-) & 0.96 & 0.98 & 0.98 & 0.99 & 1.01 & 0.99 \\
\hline & & \multicolumn{6}{|c|}{ Systems to be modelled with equivalent culvert approach } \\
\hline \multirow{6}{*}{$\begin{array}{l}\text { Upper reservoir } \\
\text { finite }(\sigma=0.325)\end{array}$} & Parameter $t_{f u l l}$ & $2 \mathrm{MC}$ & $1 \mathrm{SC}+1 \mathrm{MC}$ & $1 \mathrm{SC}+1 \mathrm{LC}$ & $1 \mathrm{MC}+1 \mathrm{LC}$ & $1 \mathrm{MC}$ with 2 ports & $1 \mathrm{LC}$ with 2 ports \\
\hline & measured (s) & 40.0 & 35.2 & 36.9 & 41.4 & 77.3 & 85.5 \\
\hline & predicted (Ib) (s) & 37.2 & 33.1 & 34.3 & 38.8 & 72.0 & 79.7 \\
\hline & predicted $(I b) /$ measured (-) & 0.93 & 0.94 & 0.93 & 0.94 & 0.93 & 0.93 \\
\hline & predicted (IIb)/measured (-) & 1.07 & 1.08 & 1.07 & 1.08 & 1.07 & 1.08 \\
\hline & predicted (16)/measured (-) & 1.38 & 1.38 & 1.37 & 1.40 & 1.30 & 1.33 \\
\hline \multirow[t]{6}{*}{ quasi infinite $(\sigma \approx 0)$} & measured (s) & 61.8 & 53.9 & 55.3 & 62.2 & 113.1 & 125.0 \\
\hline & predicted (Ib or IIb) (s) & 54.9 & 48.5 & 50.2 & 56.9 & 104.8 & 115.8 \\
\hline & predicted (Ib or IIb)/measured (-) & 0.89 & 0.90 & 0.91 & 0.91 & 0.93 & 0.93 \\
\hline & predicted (16)/measured (-) & 1.09 & 1.10 & 1.11 & 1.13 & 1.09 & 1.10 \\
\hline & predicted (LOCKSIM1)/measured (-) & 0.92 & 0.93 & 0.94 & 0.95 & 0.95 & 0.95 \\
\hline & predicted (LOCKSIM2)/measured (-) & 0.98 & 0.98 & 0.99 & 1.00 & 0.96 & 0.96 \\
\hline
\end{tabular}


Table 6 Height of first overtravel peak $\left(z_{1}-z_{e}\right)$ as predicted by analytical formula (Ic) and LOCKSIM, in comparison to the value derived from the measurements

\begin{tabular}{|c|c|c|c|c|c|c|c|}
\hline \multirow[b]{2}{*}{ Upper reservoir } & \multirow[b]{2}{*}{ Parameter $\left(z_{1}-z_{e}\right)$} & \multicolumn{6}{|c|}{ Systems for which " $n$ identical culverts" model applies } \\
\hline & & $1 \mathrm{SC}$ & $1 \mathrm{MC}$ & $2 \mathrm{MC}$ & $1 \mathrm{LC}$ & $2 \mathrm{LC}$ & $1 \mathrm{LC}$ reduced lift \\
\hline \multirow[t]{3}{*}{ finite $(\sigma=0.325)$} & measured $(\mathrm{mm})$ & - & 4.9 & 10.2 & 6.3 & 13.4 & 6.1 \\
\hline & predicted (Ic) (mm) & - & 6.0 & 11.9 & 7.1 & 14.2 & 7.1 \\
\hline & predicted (Ic)/measured (-) & - & 1.22 & 1.17 & 1.13 & 1.06 & 1.16 \\
\hline \multirow[t]{7}{*}{ quasi infinite $(\sigma \approx 0)$} & measured $(\mathrm{mm})$ & 4.1 & 4.6 & 7.3 & 5.6 & 10.6 & 6.3 \\
\hline & predicted $(\mathrm{Ic})(\mathrm{mm})$ & 5.5 & 5.9 & 11.9 & 7.1 & 14.2 & 7.1 \\
\hline & predicted (Ic)/measured (-) & 1.34 & 1.28 & 1.63 & 1.27 & 1.34 & 1.13 \\
\hline & predicted (LOCKSIM1)/measured (-) & 1.34 & 1.30 & 1.63 & 1.27 & 1.35 & 1.13 \\
\hline & predicted (LOCKSIM2)/measured (-) & 1.15 & 1.20 & 1.16 & 1.23 & 1.07 & 1.13 \\
\hline & predicted (LOCKSIM3)/measured (-) & 1.10 & 1.13 & 1.14 & 1.16 & 1.04 & 1.06 \\
\hline & & \multicolumn{6}{|c|}{ Systems to be modelled with equivalent culvert approach } \\
\hline Upper reservoir & Parameter $\left(z_{1}-z_{e}\right)$ & $2 \mathrm{MC}$ & $1 \mathrm{SC}+1 \mathrm{MC}$ & $1 \mathrm{SC}+1 \mathrm{LC}$ & $1 \mathrm{MC}+1 \mathrm{LC}$ & $1 \mathrm{MC}$ with 2 ports & $1 \mathrm{LC}$ with 2 ports \\
\hline \multirow[t]{3}{*}{ finite $(\sigma=0.325)$} & measured $(\mathrm{mm})$ & 10.2 & 11.0 & 10.9 & 11.1 & 6.9 & 7.6 \\
\hline & predicted $(\mathrm{Ic})(\mathrm{mm})$ & 11.9 & 11.2 & 11.6 & 12.8 & 7.1 & 8.2 \\
\hline & predicted (Ic)/measured (-) & 1.17 & 1.02 & 1.06 & 1.15 & 1.03 & 1.08 \\
\hline \multirow[t]{5}{*}{ quasi infinite $(\sigma \approx 0)$} & measured $(\mathrm{mm})$ & 7.3 & 9.0 & 9.8 & 9.5 & 7.0 & 7.7 \\
\hline & predicted (Ic) (mm) & 11.9 & 11.2 & 11.6 & 12.8 & 7.1 & 8.2 \\
\hline & predicted (Ic)/measured (-) & 1.63 & 1.24 & 1.18 & 1.35 & 1.01 & 1.06 \\
\hline & predicted (LOCKSIMI)/measured (-) & 1.63 & 1.26 & 1.19 & 1.36 & 1.01 & 1.06 \\
\hline & predicted (LOCKSIM2)/measured (-) & 1.14 & 1.03 & 1.04 & 1.09 & 1.11 & 1.23 \\
\hline
\end{tabular}


Table 7 Frequency of chamber surface oscillations around equalization level $\omega$ as predicted by analytical formula (Ie), in comparison to the value derived from the measurements

\begin{tabular}{|c|c|c|c|c|c|c|c|}
\hline \multirow[b]{2}{*}{ Upper reservoir } & \multirow[b]{2}{*}{ Parameter $\omega$} & \multicolumn{6}{|c|}{ Systems for which " $n$ identical culverts" model applies } \\
\hline & & $1 \mathrm{SC}$ & $1 \mathrm{MC}$ & $2 \mathrm{MC}$ & $1 \mathrm{LC}$ & $2 \mathrm{LC}$ & 1LC reduced lift \\
\hline \multirow[t]{3}{*}{ finite $(\sigma=0.325)$} & measured $\left(\mathrm{s}^{-1}\right)$ & - & 0.088 & 0.135 & 0.074 & 0.097 & 0.074 \\
\hline & predicted $(\mathrm{Ie})\left(\mathrm{s}^{-1}\right)$ & - & 0.092 & 0.130 & 0.076 & 0.108 & 0.076 \\
\hline & predicted (Ie)/measured (-) & - & 1.05 & 0.96 & 1.03 & 1.11 & 1.03 \\
\hline \multirow[t]{4}{*}{ quasi infinite $(\sigma \approx 0)$} & measured $\left(\mathrm{s}^{-1}\right)$ & 0.092 & 0.070 & 0.115 & 0.059 & 0.084 & 0.076 \\
\hline & predicted $(\mathrm{Ie})\left(\mathrm{s}^{-1}\right)$ & 0.105 & 0.080 & 0.113 & 0.066 & 0.094 & 0.066 \\
\hline & predicted (Ie)/measured (-) & 1.14 & 1.14 & 0.98 & 1.12 & 1.12 & 0.87 \\
\hline & & \multicolumn{6}{|c|}{ Systems to be modelled with equivalent culvert approach } \\
\hline Upper reservoir & Parameter $\omega$ & $2 \mathrm{MC}$ & $1 \mathrm{SC}+1 \mathrm{MC}$ & $1 \mathrm{SC}+1 \mathrm{LC}$ & $1 \mathrm{MC}+1 \mathrm{LC}$ & $1 \mathrm{MC}$ with 2 ports & $1 \mathrm{LC}$ with 2 ports \\
\hline \multirow[t]{3}{*}{ finite $(\sigma=0.325)$} & measured $\left(\mathrm{s}^{-1}\right)$ & 0.135 & 0.125 & 0.123 & 0.122 & 0.071 & 0.068 \\
\hline & predicted $(\mathrm{Ie})\left(\mathrm{s}^{-1}\right)$ & 0.130 & 0.152 & 0.143 & 0.119 & 0.092 & 0.076 \\
\hline & predicted (Ie)/measured (-) & 0.96 & 1.22 & 1.16 & 0.98 & 1.30 & 1.12 \\
\hline \multirow[t]{3}{*}{ quasi infinite $(\sigma \approx 0)$} & measured $\left(\mathrm{s}^{-1}\right)$ & 0.115 & 0.110 & 0.116 & 0.096 & 0.064 & 0.057 \\
\hline & predicted $(\mathrm{Ie})\left(\mathrm{s}^{-1}\right)$ & 0.113 & 0.132 & 0.124 & 0.104 & 0.080 & 0.066 \\
\hline & predicted (Ie)/measured (-) & 0.98 & 1.20 & 1.07 & 1.08 & 1.25 & 1.16 \\
\hline
\end{tabular}




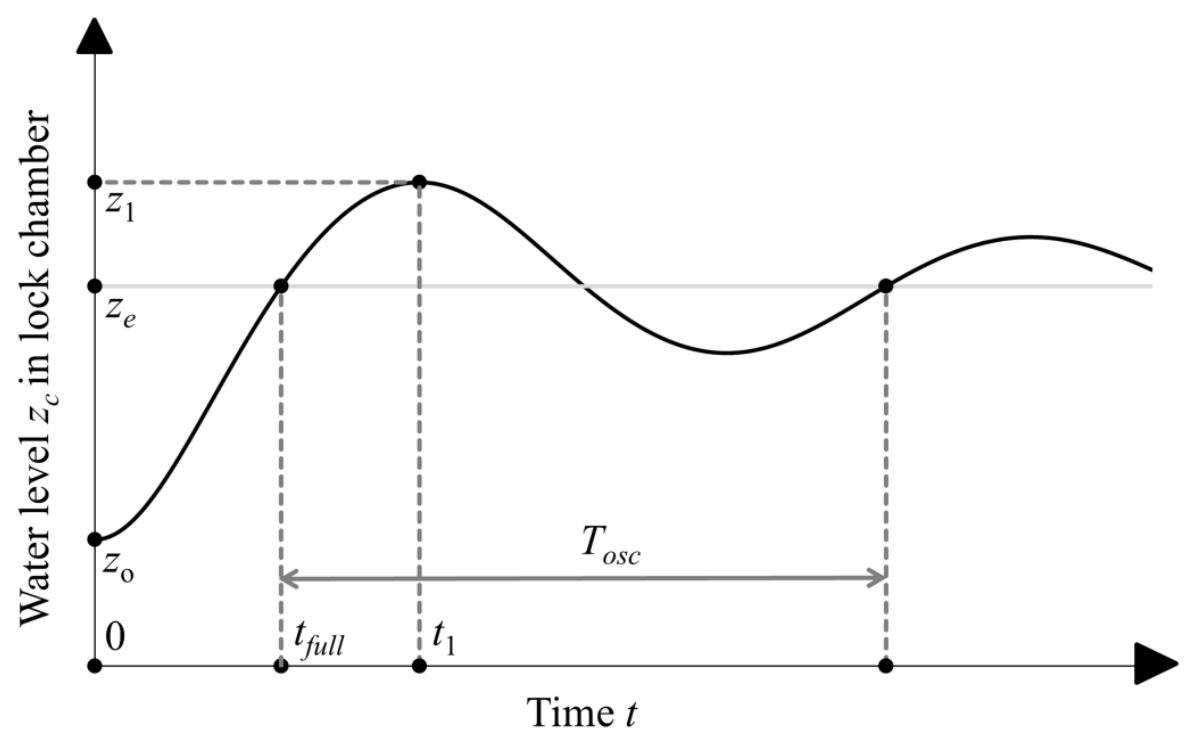

Figure 1 The overtravel phenomenon and its main parameters: the initial level $z_{0}$, the filling time $t_{f u l l}$, the water level $z_{1}$ and the time $t_{1}$ of the first overtravel peak, the time interval $T_{\text {osc }}$ between first two up-crossings of equalization level $z_{e}$

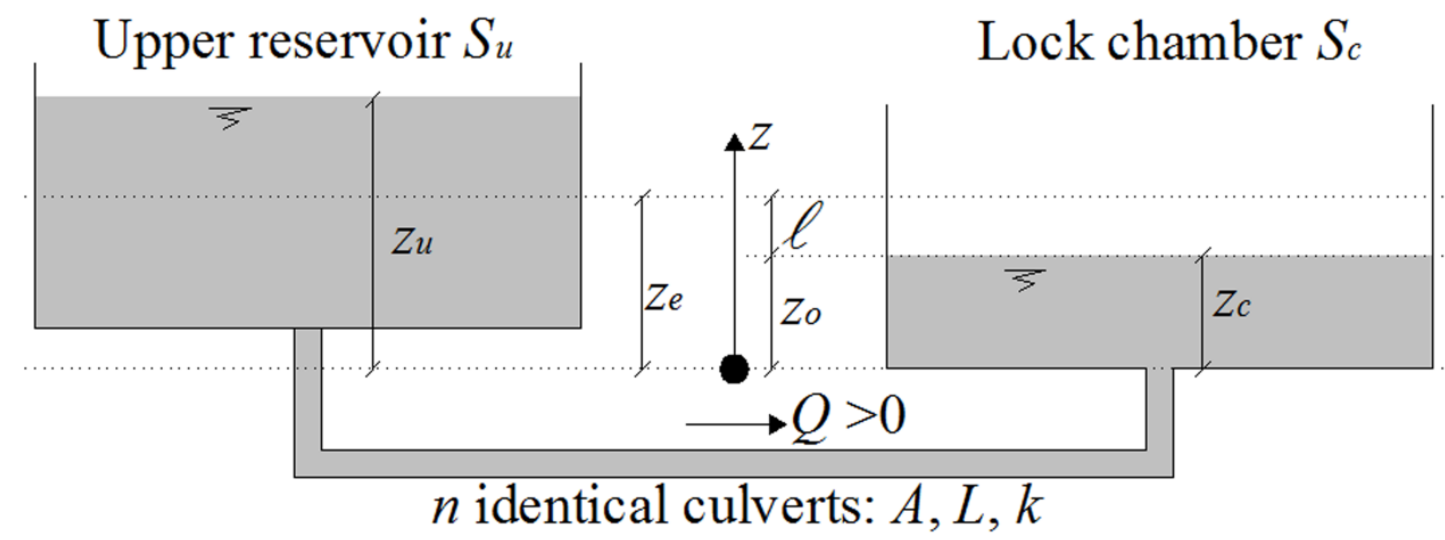

Figure 2 Definition sketch for navigation lock filling with $n$ identical culverts 

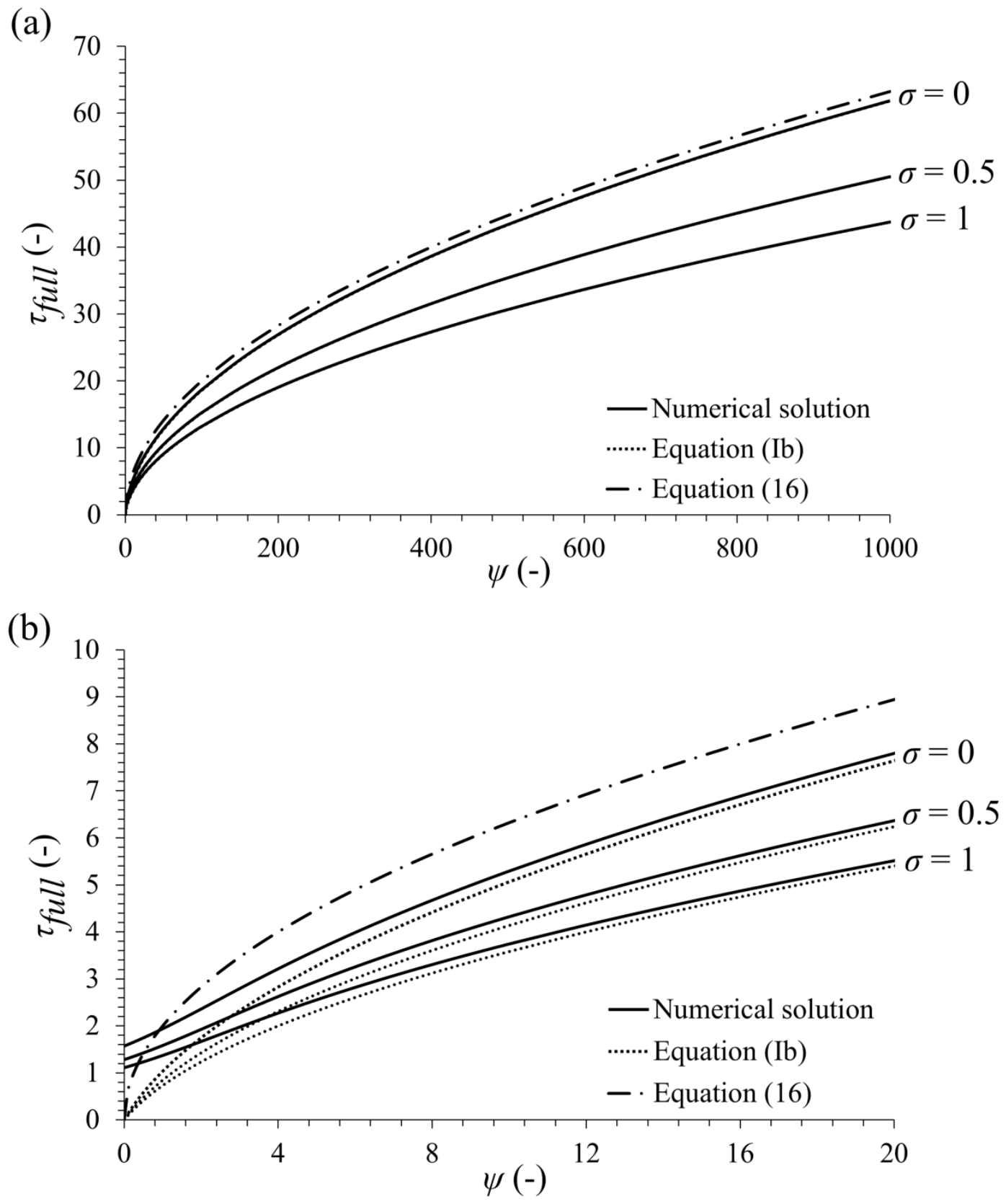

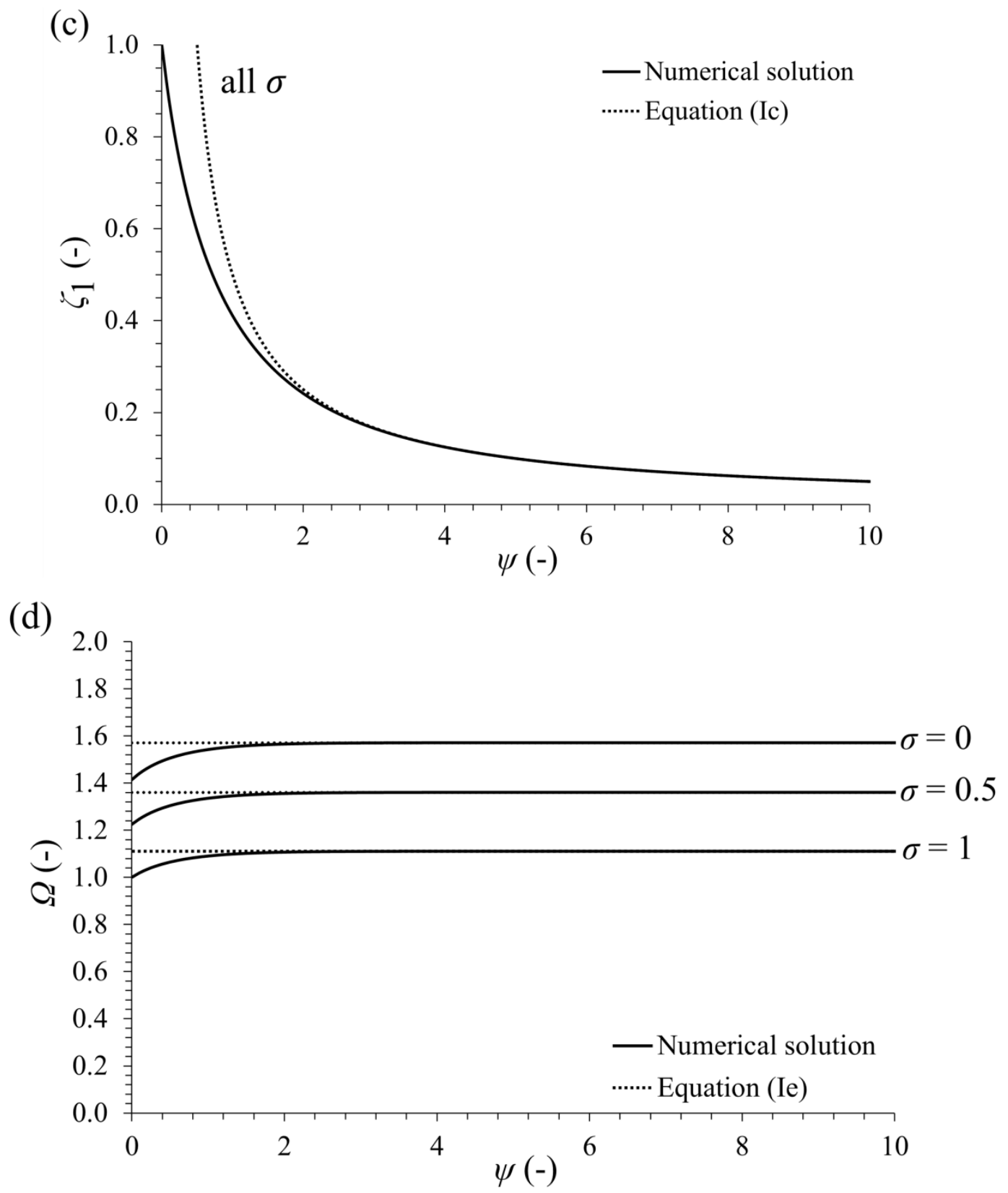

Figure 3 Comparison of analytical formulae to accurate numerical solutions of the complete equation, for different values of parameters $\psi$ and $\sigma$. Figures $3 \mathrm{a}$ and $3 \mathrm{~b}$ : filling time, $\tau_{\text {full }}$; Fig. 3c: water surface deviation from equalization level at the first overtravel peak, $\zeta_{1}$; Fig. 3d: frequency of chamber surface oscillations around equalization level, $\Omega$ 


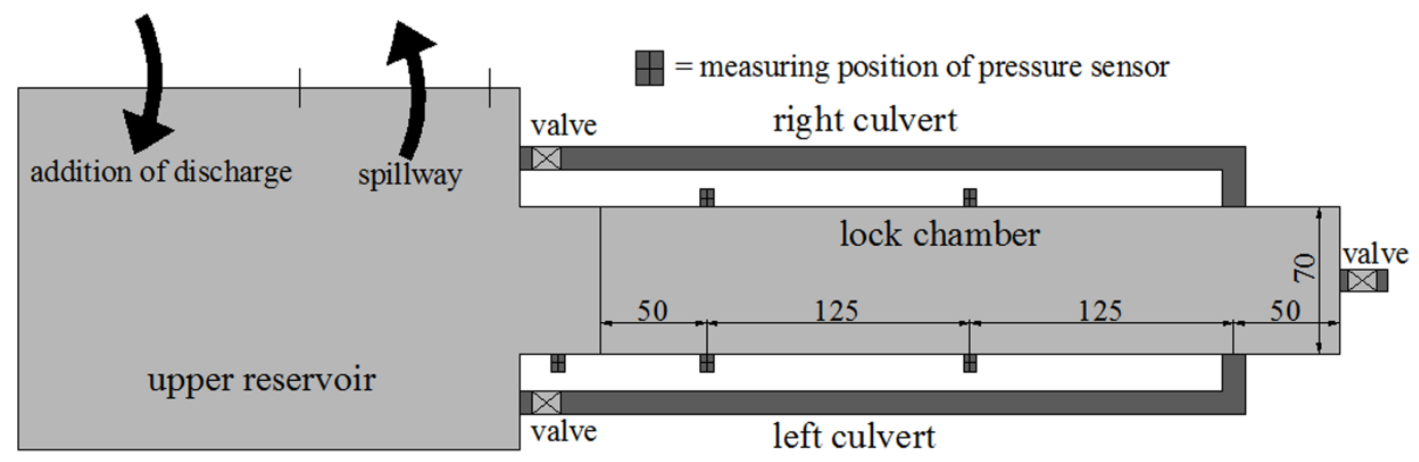

Figure 4 Plan view of experimental set-up, dimensions in $\mathrm{cm}$

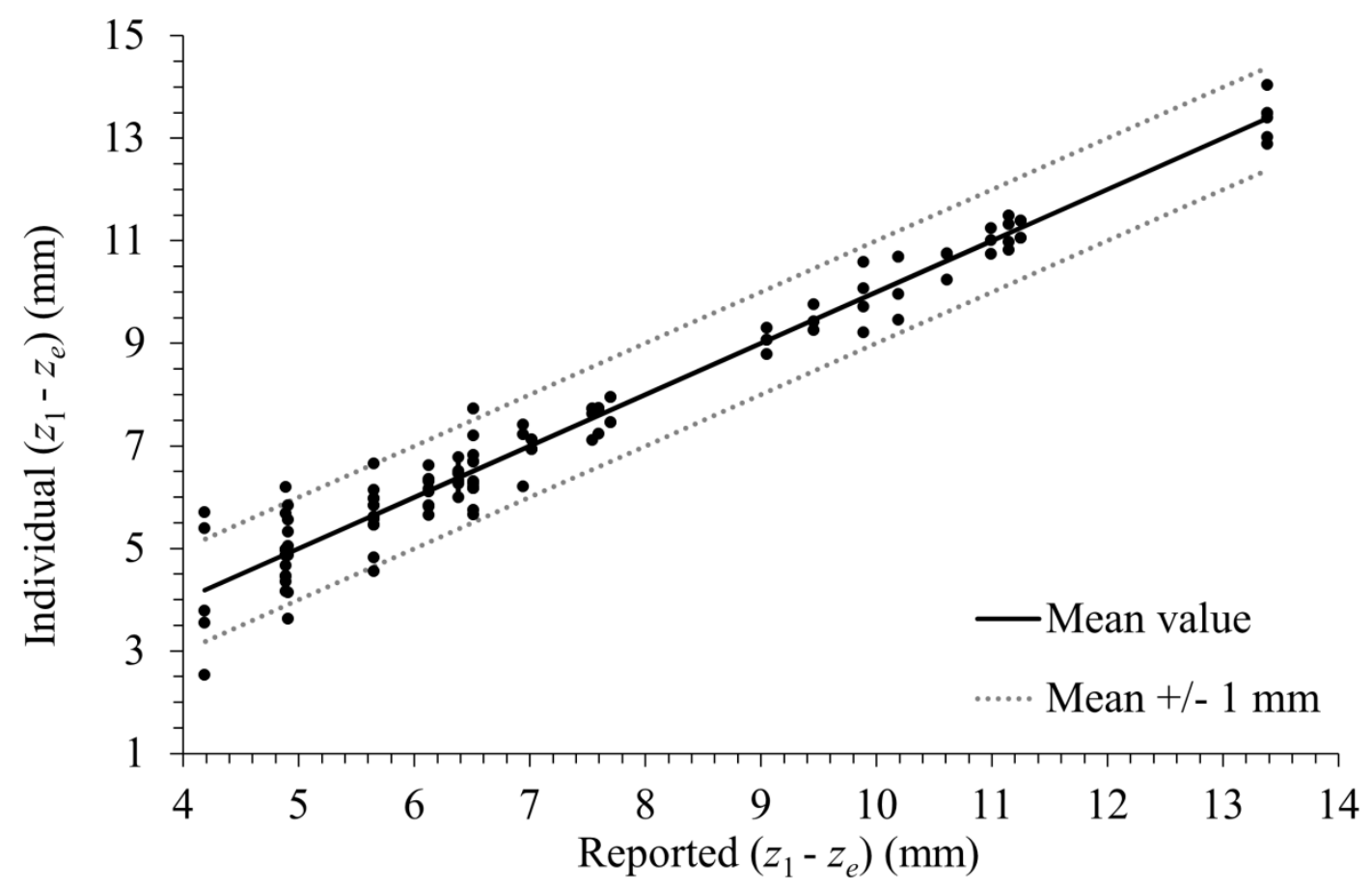

Figure 5 Individual measurements versus reported values for $\left(z_{1}-z_{e}\right)$ 